\title{
Traumatic Partial Aniridia and Cataract after Iris-Fixated Foldable Phakic Intraocular Lens Implantation
}

\author{
Joo Hyun Jin Kwon Chung Sung Jin Lee \\ Department of Ophthalmology, Soonchunhyang University, College of Medicine, \\ Seoul, Republic of Korea
}

\section{Key Words}

Artiflex phakic intraocular lens · Artisan phakic intraocular lens · Phakic intraocular lens ·

Traumatic aniridia

\begin{abstract}
We present the first case of partial aniridia, traumatic cataract, and peripapillary subretinal hemorrhage after blunt trauma to an eye containing a foldable iris-fixated phakic intraocular lens (pIOL). Although the patient experienced visual loss because of considerable corneal astigmatism and a traumatic cataract, vision was recovered by a 2-stage primary corneal repair and cataract surgery with a toric intraocular lens. This case exhibits clinical manifestations that differ from previously reported trauma of a non-foldable iris-fixated pIOL. A relatively small corneal incision and a large optic diameter of Artiflex pIOLs may prevent posttraumatic total expulsion of intraocular tissues and present different clinical features from the case of non-foldable pIOLs (Artisan). Although the extent of the damage is different, the possibility of aniridia and traumatic cataract exists in eyes with both foldable and non-foldable iris-fixated pIOLs.

(C) 2013 S. Karger AG, Basel
\end{abstract}

\section{Introduction}

Phakic intraocular lenses (pIOLs) are generally accepted as safe and effective treatment options for the correction of moderate to high myopia. The Artiflex pIOL (Ophtec BV, Groningen, The Netherlands), a foldable version of the Artisan pIOL (anterior chamber irisfixated pIOL), has a flexible polysilicon optic and 2 rigid polymethylmethacrylate haptics [1]. The foldability of the Artiflex pIOL allows a smaller incision than that required for the Artisan pIOL and normally does not require sutures. These advantages can provide faster 
Hyun et al.: Traumatic Partial Aniridia and Cataract after Iris-Fixated Foldable Phakic Intraocular Lens Implantation

visual recovery and better uncorrected visual acuity than the Artisan pIOL [2]. It can also be assumed that Artiflex pIOL implantation is safer with respect to blunt trauma than Artisan pIOL implantation because of its flexible optical portion and the small corneal incision required, which is similar to that used in modern cataract surgery.

Although trauma can dislocate an iris-fixated pIOL, no previous report has been issued of a serious complication after trauma in a patient with an Artiflex pIOL [3]. Here, we present a case of traumatic partial aniridia, cataract, and peripapillary subretinal hemorrhage and its management after Artiflex pIOL implantation. Furthermore, we present different patterns of damage between the eyes with Artiflex and Artisan pIOLs.

\section{Case Report}

A 27-year-old woman presented with a sudden loss of vision and ocular pain in her left eye after blunt trauma with a hand during a quarrel. The patient had a history of bilateral implantation of an Artiflex pIOL 1 month previously to correct -6.5 diopters (D) of myopia. On initial examination of the left eye, visual acuity was hand motion and the eye had a 6-mm corneoscleral laceration extending from the previous superior clear corneal incision. The iris was dialyzed from 7 to 1 o'clock, and half had prolapsed along with the medial haptic side grasping the iris through the wound; the haptic-optic junction was stuck in the wound (fig. $1 \mathrm{a}, \mathrm{b})$. The optic and another haptic enclavated on the iris stroma of the pIOL were left in the anterior chamber, and partial hyphema was also present. The absence of retinal detachment and vitreous hemorrhage was confirmed by B-scan ultrasonography.

After removing the firmly enclavated pIOL, the prolapsed iris was repositioned with partial resection of the severely damaged iris. The corneoscleral laceration was repaired with 10-0 nylon with interrupted suture (fig. 1c). Hyphema and inflammation subsequently subsided, and then a traumatic cataract and a peripapillary subretinal hemorrhage 1 disc diameter in size were found (fig. 1d).

Three weeks after surgery, the corneoscleral sutures were removed. The best-corrected visual acuity was $20 / 40$ with $-3.75-4.0 \times 130$, and the intraocular pressure was normal. Corneal topography showed $4.0 \mathrm{D}$ astigmatism at 40 degrees, and the corneal endothelial cell count was 1,967 cells $/ \mathrm{mm}^{2}$. The peripapillary subretinal hemorrhage resolved spontaneously (fig. 2a).

Six weeks after primary repair, the traumatic cataract had become denser, and partial aniridia with iridodialysis remained (fig. 2b). Partial zonular dialysis from 8 to 10 o'clock was noticed. No further change in corneal astigmatism was confirmed by 3 consecutive topographies conducted at 1-month intervals.

Five months after presentation, the traumatic cataract was removed, and a toric intraocular lens (Acrysof Toric IOL, model SN60T5; Alcon Laboratories, Inc., Fort Worth, Tex., USA; models T6-T9 were not available in Korea) was implanted to correct the large amount of astigmatism. A clear corneal incision was made on the steepest axis of the cornea, and a capsular tension ring was used for partial zonular dialysis. The iridodialysis was repaired with mattress sutures with 10-0 polypropylene (fig. 2c).

Six months after the second surgery, the uncorrected visual acuity of the left eye was $20 / 32$ and the best-corrected visual acuity was $20 / 20$ with plano $-1.0 \times 140$. Corneal endothelial cells had decreased to 1,634 cells $/ \mathrm{mm}^{2}$ but remained stable by serial examination, and the intraocular pressure was normal. Although partial aniridia remained, the patient experienced no discomfort during daily activities (fig. 2d). 
Hyun et al.: Traumatic Partial Aniridia and Cataract after Iris-Fixated Foldable Phakic Intraocular Lens Implantation

\section{Discussion}

In 2007, Lee [4] reported the first case of traumatic aniridia and aphakia of an eye with an Artisan pIOL. There have been many cases of trauma in patients after pIOL implantation. However, most of these cases were not severe and could be reenclavated into position.

Based on previous reports and experiences, the damage caused by blunt trauma seems to be dependent on incision size. In the present case, the iris and pIOL complex were not extruded completely and stuck in the dehisced wound. Contrary to this case, total extrusion of iris, pIOL, and crystalline lens complex was reported in a patient with a rigid Artisan pIOL with a larger incision $(6.0 \mathrm{~mm})$ [4]. Ball et al. [5] postulated that a small surgical incision serves as a 'release valve' during blunt trauma that helps avoid devastating damage, but which causes rapid expulsion of intraocular tissues due to the velocity of aqueous flow through a small incision. Although there are some differences in surgical procedure, traumatic wound dehiscence after cataract surgery is significantly less for a small incision. Ball and McLeod [6] reported that the frequency of traumatic wound dehiscence after cataract surgery was significantly less for a small sclerocorneal incision $(0.02 \%)$ than a large incision $(0.4 \%)$. Although the rate of traumatic wound dehiscence would be lower, this case suggests that major trauma can also result in eyeball rupture and severe damage, despite a small incision (3.2 mm).

The Artiflex pIOL-treated eye showed different patterns of damage from the eye treated with an Artisan pIOL after blunt trauma. These differences seem to be mainly related to the size of the incision. The large incision, in the Artisan-treated eye, encourages the devastating damage from blunt trauma. However, in Artiflex-treated eyes, the smaller incision prevents total expulsion of intraocular tissues because the Artiflex lens has a relatively large optic and it blocks the exit from the small wound.

\section{Conclusion}

Iris-fixated pIOLs are sufficient to hold the iris during trauma and result in damage to the iris and the crystalline lens. Even if the extent of the damage were different after trauma, the possibility of aniridia and traumatic cataract should be considered possible complications in eyes with both foldable and non-foldable iris-fixated pIOLs.

\section{Acknowledgment}

This work was supported by the Soonchunhyang University Research Fund.

\section{Disclosure Statement}

The authors report no potential conflict of interest relevant to this article. 
Hyun et al.: Traumatic Partial Aniridia and Cataract after Iris-Fixated Foldable Phakic Intraocular Lens Implantation

\section{References}

1 Tehrani M, Dick HB: Short-term follow-up after implantation of a foldable iris-fixated intraocular lens in phakic eyes. Ophthalmology 2005;112:2189-2195.

-2 Coullet J, Guell JL, Fournie P, Grandjean H, Gaytan J, Arne JL, Malecaze F: Iris-supported phakic lenses (rigid vs. foldable version) for treating moderately high myopia: randomized paired eye comparison. Am J Ophthalmology 2006;142:909-916.

-3 Dick HB, Budo C, Malecaze F, Guell JL, Marinho AA, Nuijts RM, Luyten GP, Menezo JL, Kohnen T: Foldable Artiflex phakic intraocular lens for the correction of myopia: two-year follow-up results of a prospective European multicenter study. Ophthalmology 2009;116:671-677.

$\rightarrow 4$ Lee SJ: Traumatic aniridia and aphakia after Artisan intraocular lens implantation. J Cataract Refract Surg 2007;33:1341-1342.

5 Ball J, Caesar R, Choudhuri D: Mystery of the vanishing iris. J Cataract Refract Surg 2002;28:180-181.

6 Ball JL, McLeod BK: Traumatic wound dehiscence following cataract surgery: a thing of the past? Eye 2001;15:42-44.

This study was presented at the 107th Korean Academy of Ophthalmology Annual Meeting on April 13, 2012 (Busan, Republic of Korea). 
Hyun et al.: Traumatic Partial Aniridia and Cataract after Iris-Fixated Foldable Phakic Intraocular Lens Implantation
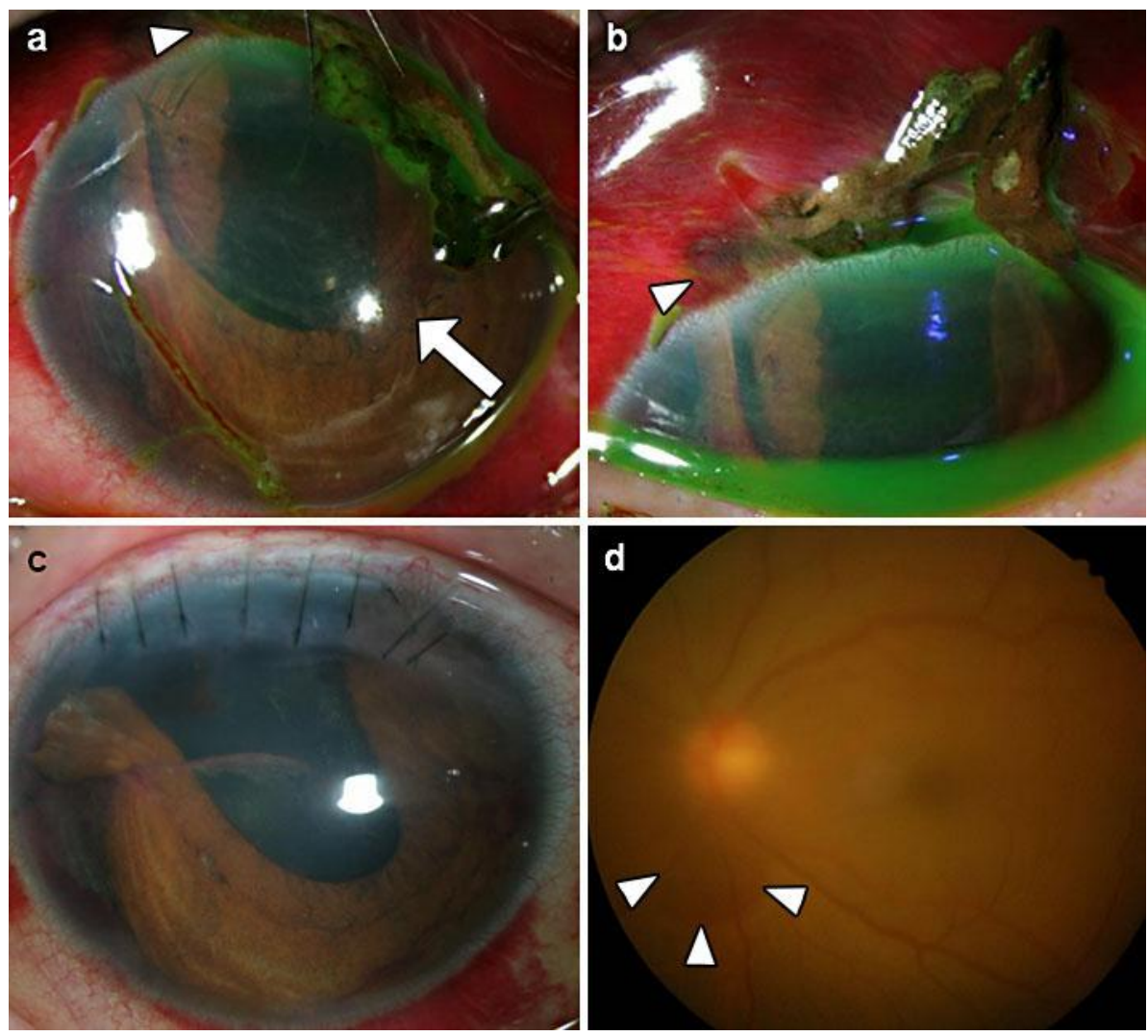

Fig. 1. Anterior segment and fundus photographs of the left eye. a At initial presentation, the dialyzed iris and the medial haptic of the pIOL were prolapsed with superior wound dehiscence (arrowhead). The Artiflex pIOL was dislocated in the anterior chamber, and another haptic was enclavated on the temporal iris (arrow). b Iridodialysis at 7 to 1 o'clock and prolapse of the iris and 1 of the haptics through the wound; the haptic-optic junction was stuck to the wound (arrowhead). c Slit-lamp photograph after primary repair. Partial aniridia at 7 to 1 o'clock with iridodialysis remained. d Fundus photography shows an inferior peripapillary subretinal hemorrhage 1 disc diameter in size (arrowheads). 
Case Reports in

Ophthalmology

\begin{tabular}{l|l}
\hline Case Rep Ophthalmol 2013;4:210-215 \\
\hline DOI: 10.1159/000356239 & $\begin{array}{l}\text { @ 2013 S. Karger AG, Basel } \\
\text { www.karger.com/cop }\end{array}$ \\
\hline
\end{tabular}

Hyun et al.: Traumatic Partial Aniridia and Cataract after Iris-Fixated Foldable Phakic Intraocular Lens Implantation
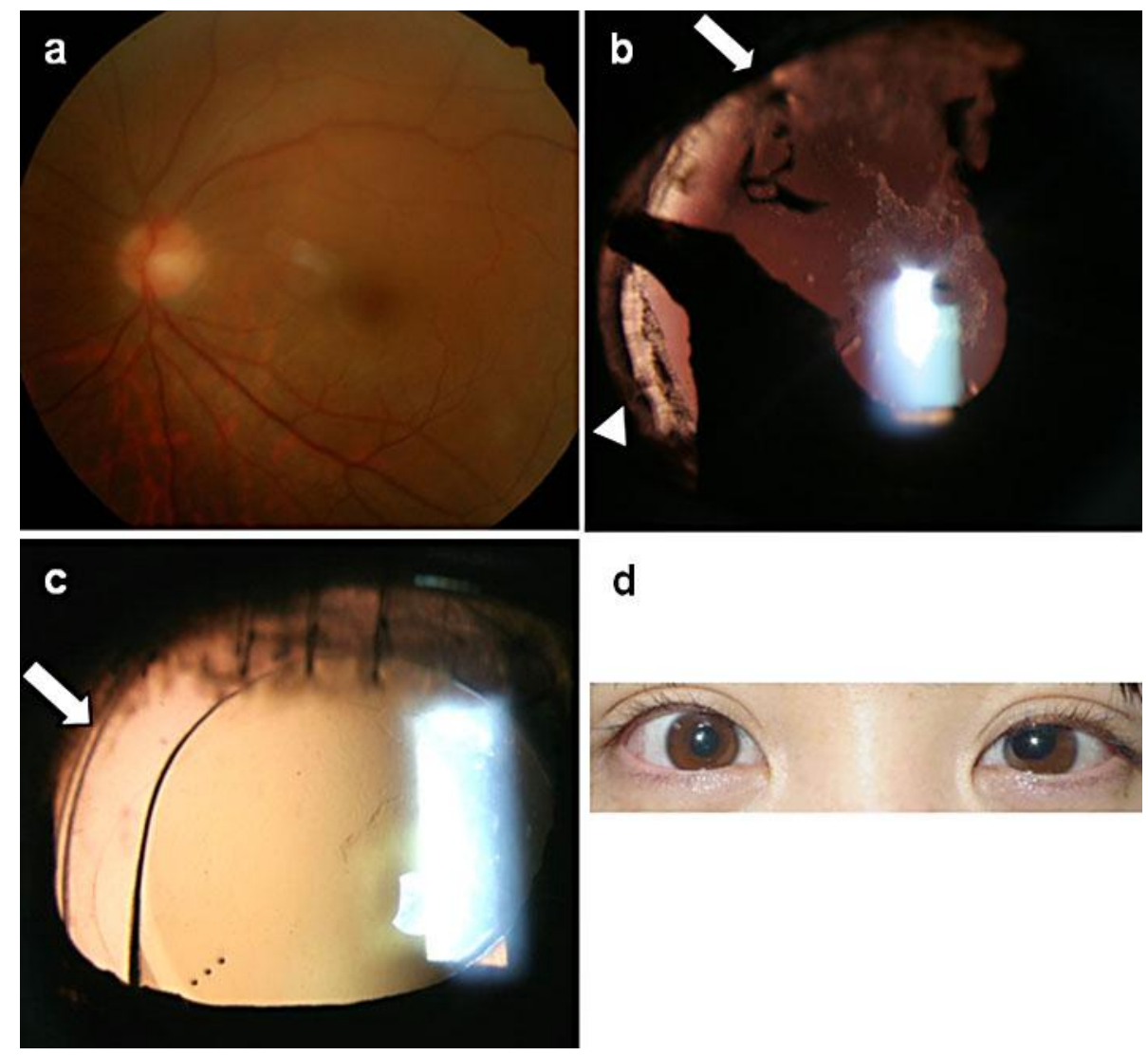

d

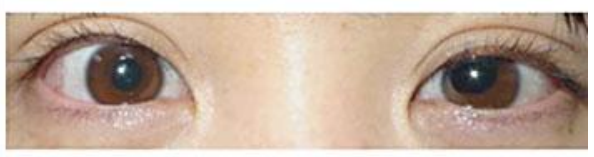

Fig. 2. Photographs after the first and second operation. a Three weeks after presentation, the peripapillary subretinal hemorrhage had resolved. $\mathbf{b}$ Six weeks after blunt trauma, the traumatic posterior subcapsular cataract at the center of the lens had become denser, zonular dialysis (arrowhead) and an iris defect were found (arrow). c Five months after initial presentation, cataract extraction with toric IOL implantation and iridoplasty were performed. A capsular tension ring (arrow) was used for zonular dialysis. $\mathbf{d}$ Six months after the second operation, partial aniridia at the superior side of the left eye was partially covered by the upper eyelid. 